\title{
Escape distribution for an inclined billiard
}

\author{
Alan Roy $^{1, *}$ and Nikolaos Georgakarakos ${ }^{2, \dagger}$ \\ ${ }^{1}$ University of Southampton, Electrical Power Engineering \\ Research Group,ECS, Highfield, Southampton, UK \\ ${ }^{2} 128$ V. Olgas str., Thessaloniki 54645, Greece
}

\begin{abstract}
Hénon [8] used an inclined billiard to investigate aspects of chaotic scattering which occur in satellite encounters and in other situations. His model consisted of a piecewise mapping which described the motion of a point particle bouncing elastically on two disks. A one parameter family of orbits, named h-orbits, was obtained by starting the particle at rest from a given height. We obtain an analytical expression for the escape distribution of the h-orbits, which is also compared with results from numerical simulations. Finally, some discussion is made about possible applications of the h-orbits in connection with Hill's problem.
\end{abstract}

MSC2010 numbers: 37D45 • 37D50 • 70B05 • 70F07

Keywords: Chaotic scattering, inclined billiards, Hill's problem

\section{INTRODUCTION}

Chaotic scattering is a phenomenon which appears in many scientific fields, such as astronomy, electromagnetism, statistical mechanics, chemistry, quantum mechanics, just to mention a few. Two objects that are separated initially by some distance, come closer, interact with each other for a while and then they separate again.

More specifically, in astronomy, chaotic scattering can be found in many situations, such as, for example, in the formation and evolution of the outer solar system (e.g. [1]), in planetary rings (e.g. [11]), in exosolar planetary systems (e.g. [4]), in the dynamical evolution

*Electronic address: A.A.Roy@soton.ac.uk

$\dagger$ Electronic address: georgakarakos@hotmail.com 
of globular clusters (e.g. [3]). Regarding the last situation, stars are expected to escape from a globular cluster due to a variety of dynamical processes, such as two body relaxation or an external tidal field. This theoretical prediction has also been confirmed by observations (e.g. [9]). It is important to know whether a star will escape from a cluster and also the timescale on which that will happen as it affects the scaling of the N-body simulation results (normally, the number $\mathrm{N}$ of stars used by a simulation is less than the number of stars of the cluster under study) and reduce the computational effort required to perform a simulation. Also, the timescale of escape is important when one uses Monte Carlo models in order to study the dynamical evolution of globular clusters. Further reasons regarding the importance of escape in globular clusters are discussed in [6].

A star can escape from a cluster when its energy exceeds some critical threshold but escape may not occur immediately. Numerical simulations show that it may take a long time for a star to escape or it may not even escape at all (escape on a timescale comparable with the age of the universe falls into this category too). The assumption of rapid escape referred to in [5] and [2] is therefore a significant source of error for theoretical predictions regarding the evolution of globular clusters. Interest has therefore been generated in the dynamics underpinning the escape mechanism.

Petit and Hénon [10] investigated numerically the motion of two satellites around a planet. However, certain difficulties arose in their study and in order to overcome those difficulties, Hénon [8] devised a model problem which demonstrated similar behaviour to the satellite problem but it was easier to study. That model consisted of an inclined billiard, i.e. a point particle bouncing on two fixed disks and a two dimensional mapping was used to describe the dynamics of the model.

In the present paper, we extend the results obtained in [8] by deriving an analytical expression for the escape distribution of a particular class of orbits, the so called h-orbits. The structure of the paper is roughly as follows: first, we give a description of the model developed by Hénon (Sec. 2). Then we obtain an analytical expression for the escape distribution of the h-orbits and the formula is compared with results from numerical simulations (Sec. 3). Next, there is some discussion about the billiard model and Hill's problem, which originally inspired Hénon to devise the inclined billiard model (Sec. 4). Finally, the last section of the paper gives a brief summary. 


\section{INCLINED BILLIARD MODEL}

Hénon's model is described as follows: a point particle moves in the (X,Y) plane and bounces elastically on two fixed disks of radius $r$ whose centres are located at $(-1,-r)$ and $(1,-r)$. In addition, the particle is subject to a constant acceleration $g$ in the negative $Y$ direction. For reasons of simplicity, $r$ is considered to be large, an assumption which means that the two disks overlap.

A one parameter family of orbits, the so called h-orbits, is defined by assuming that the particle is dropped from rest at $\left(h, Y_{0}\right) . Y_{0}$ is a positive constant which fixes the energy and $h$ is a variable. For those orbits, there are intervals of $h$ in which the orbit changes continuously and there are also critical values of $h$ at which a transition in behaviour is observed. For $h=1$ the particle bounces ad infinitum on the right disk, while for $h=-1$ the particle bounces ad infinitum on the left disk. If the particle is dropped either to the left of the left disk or to the right of the right disk, then the particle escapes and never returns. If the particle is dropped either to the right of the left disk or to the left of the right disk, then the particle exhibits a more complex behaviour, bouncing from one disk to another. Hence, the values $h= \pm 1$ are associated with a transition. In general, transitions are present at values of $h$ which lead to solutions which approach asymptotically one of the $h= \pm 1$ orbits.

A convenient way of dealing with the problem is to introduce a two dimensional explicit mapping, which is equivalent to studying a Hamiltonian system with two degrees of freedom. Details about the mapping and its properties can be found in [8]. If $\left(X_{j}, Y_{j}\right)$ is the position of the particle on the $j$ 'th rebound, then the two dimensional mapping, keeping the notation of [8], is the following:

$$
\begin{aligned}
& X_{j+1}=X_{j} \cosh \phi+w_{j} \sinh \phi-s_{j}(\cosh \phi-1) \\
& w_{j+1}=X_{j} \sinh \phi+w_{j} \cosh \phi-\left(s_{j} \cosh \phi+s_{j+1}\right) \tanh \frac{\phi}{2} .
\end{aligned}
$$

$w_{j}$ is defined by

$$
W_{j}=\frac{w_{j} g}{2 \sqrt{2 E}} \sinh \phi,
$$

where $W_{j}$ is the transverse velocity, $E$ is the total energy and $\phi$ is a dimensionless parameter 
defined by

$$
\cosh \phi=1+\frac{4 E}{g r}, \quad \sinh \phi=\sqrt{\frac{4 E}{g r}\left(2+\frac{4 E}{g r}\right)} .
$$

Finally, $s_{j}=\operatorname{sign} X_{j}$. If the mapping is applied repeatedly, we obtain:

$$
\begin{aligned}
X_{n} & =\frac{h \cosh \left[\left(n-\frac{1}{2}\right) \phi\right]}{\cosh \frac{\phi}{2}}-2 \tanh \frac{\phi}{2} \sum_{j=1}^{n-1} s_{j} \sinh [(n-j) \phi], \\
w_{n} & =\frac{h \sinh \left[\left(n-\frac{1}{2}\right) \phi\right]}{\cosh \frac{\phi}{2}}-2 \tanh \frac{\phi}{2} \sum_{j=1}^{n-1} s_{j} \sinh [(n-j) \phi]-s_{n} \tanh \frac{\phi}{2} .
\end{aligned}
$$

A given h-orbit is associated to a sequence of binary digits ( 0 or 1 depending on whether the particle bounces on the left or right disk respectively) and the corresponding to that sequence number $\mathrm{A}$, with $0 \leq A \leq 1$. An h-orbit changes continuously within an interval of continuity. In that interval, the sequence of rebounds remains the same throughout the whole of this interval. This means that the value of $\mathrm{A}$ is constant. If $\mathrm{A}$ is plotted as a function of $\mathrm{h}$ (for a fixed value of $\phi$ ) a fractal picture is generated. This has the appearance of the Devil's staircase, which consinsts of an infinite number of horizontal bars. Each bar corresponds to an interval of continuity, meaning that when a particle starts within that interval, it will follow the same orbit in terms of which disk it bounces on. Such an example is given in fig. 1 .

\section{THE ESCAPE DISTRIBUTION}

\section{A. Analytical derivation}

Now, we are going to obtain an expression for the number of bounces it takes for an h-orbit to escape from the system. The derivation will apply for $e^{\phi} \leq \frac{1}{3}$ or $e^{\phi} \geq 3$. When $\frac{1}{3}<e^{\phi}<3$, the Devil's staircase is not continuous any longer and gaps appear between the horizontal bars, i.e. there exist values of $A$ to which no h-orbits correspond. Also, many of the assumptions made about the h-orbits do not hold. More details about that situation can be found in [8], although, for reasons not known to us, Hénon only refers to properties regarding $e^{\phi}=3$. The mapping given by Eqs (1) and (2) has two fixed points $(X=-1, w=0$ and $X=1, w=0)$, each one with eigenvalues $e^{\phi}$ and $e^{-\phi}$, which suggests that when $e^{\phi} \geq 3$, we get at the same time $e^{-\phi} \leq \frac{1}{3}$. For $e^{\phi}=1$, all the points of the mapping 
are fixed points. However, as stated in [8], it is not clear whether the peculiarities of the $\frac{1}{3}<e^{\phi}<3$ case have general relevance to the problem of chaotic scattering.

The time to escape, for a given value of $\mathrm{h}$, is the smallest integer $k$ such that $\left|X_{n}\right|>1$ for all $n>k$. In particular, we would like to know which values of h, i.e. which sub-intervals of $(-1,1)$, correspond to orbits which escape after at least $k$ bounces within the constrained region $(-1 \leq X \leq 1)$. By summing up the lengths of these sub-intervals it is possible to produce an escape distribution.

According to [8], at each end of every horizontal bar there are left and right asymptotic orbits corresponding to

$$
h_{+}=\left(e^{\phi}-1\right) \sum_{j=1}^{p-1} e^{-j \phi} s_{j}+\left(e^{\phi}-2\right) e^{-p \phi},
$$

and

$$
h_{-}=\left(e^{\phi}-1\right) \sum_{j=1}^{p-1} e^{-j \phi} s_{j}-\left(e^{\phi}-2\right) e^{-p \phi},
$$

respectively, where $p$ is associated with each horizontal bar (p-bar), indicating the number of digits which occurred in the binary sequence before the repetition of either zeros or ones, i.e. before the particle escapes over the left or right disk. Those orbits take infinitely many bounces to escape. If we move away from those extremes and consider $h=h_{+}-\delta$ or $h=h_{-}+\delta$, we find orbits which escape after a finite number of bounces. The smaller $\delta$ is, the longer it takes for an orbit to escape (more precisely, $k \rightarrow \infty$ as $\delta \rightarrow 0$ ). For values of $\mathrm{h}$ in the interval $\left(h_{-}, h_{+}\right)$, for a given p-bar, the number of bounces never falls below $\mathrm{p}$.

In order to find those intervals of $h$ which correspond to orbits that remain bound for at least $k$ bounces, we require to sum together the full lengths of the horizontal bars for which $p \geq k$. Only partial contributions will be required from the bars with $p<k$. This is because some values of $h$ near the centre of these bars lead to orbits which escape too rapidly (under $k$ bounces). The whole calculation may be performed in the following three steps:

i) calculating the partial contribution from a bar with $p<k$ by finding an expression for the time to escape, $k$, as a function of $\delta$. This expression may be inverted to yield $\delta$ as a function of $k$. We refer to $\delta_{r}$ or $\delta_{l}$ depending on which end is being considered. The total partial contribution from each bar is given by $\delta_{r}+\delta_{l}$.

ii) summing together the partial contributions (of which there are $2^{p-1}$ ) from all the p-bars for each $p<k$.

iii) considering the contributions from all bars with $p \geq k$ (the length of a bar is $h_{+}-h_{-}$). 
We start with the first step, calculating an expression for $k(\delta)$ by considering the values of h which lead to left and right asymptotic orbits. Substituting Eq. (4) into Eq. (3), we obtain

$$
\begin{aligned}
X_{n} & =\left[\left(e^{\phi}-1\right) \sum_{j=1}^{p-1} e^{-j \phi} s_{j}+\left(e^{\phi}-2\right) e^{-p \phi}\right] \frac{\cosh \left[\left(n-\frac{1}{2}\right) \phi\right]}{\cosh \frac{\phi}{2}}- \\
& -2 \tanh \frac{\phi}{2} \sum_{j=1}^{n-1} s_{j} \sinh [(n-j) \phi] .
\end{aligned}
$$

If we expand the above equation (keep in mind that $s_{p}=1$ and $s_{j}=-1$ for all $j \geq p+1$ since we deal with a left escaping orbit), we end up with

$$
X_{n}=-1+e^{-n \phi} L_{l}, \quad n \geq p+1
$$

where

$$
L_{l}=\frac{e^{\phi}\left(e^{\phi}-2\right) e^{-p \phi}}{e^{\phi}+1}+\frac{e^{(p+1) \phi}}{e^{\phi}+1}+\tanh \frac{\phi}{2}\left[e^{p \phi}+e^{\phi} \sum_{j=1}^{p-1} e^{-j \phi} s_{j}+\sum_{j=1}^{p-1} e^{j \phi} s_{j}\right] .
$$

Now suppose that $h=h_{+}-\delta_{l}$ and that the $s_{j}$ 's are the same as in Eq. (4), i.e we are looking at the same bar. Then, from (3), we get

$$
\begin{aligned}
X_{n}^{\prime} & =\left(h_{+}-\delta_{l}\right) \frac{\cosh \left[\left(n-\frac{1}{2}\right) \phi\right]}{\cosh \frac{\phi}{2}}-2 \tanh \frac{\phi}{2} \sum_{j=1}^{n-1} s_{j} \sinh [(n-j) \phi]= \\
& =X_{n}-\delta_{l} \frac{\cosh \left[\left(n-\frac{1}{2}\right) \phi\right]}{\cosh \frac{\phi}{2}}=-1+e^{-n \phi} L_{l}-\delta_{l} \frac{\cosh \left[\left(n-\frac{1}{2}\right) \phi\right]}{\cosh \frac{\phi}{2}} .
\end{aligned}
$$

As we are interested in the number of bounces $k$ before the particle escapes over the left disk, Eq. (5) yields $\left(X_{k}^{\prime}=-1\right)$

$$
\delta_{l}=\frac{2 e^{\frac{\phi}{2}} \cosh \frac{\phi}{2} L_{l}}{e^{2 k \phi}+e^{\phi}}=\frac{\left(e^{\phi}+1\right) L_{l}}{e^{2 k \phi}+e^{\phi}} .
$$

Following the above approach, we can obtain a similar expression for right asymptotic orbits. In this case, we find that

$$
X_{n}=1-e^{-n \phi} L_{r}, \quad n \geq p+1
$$

where

$$
L_{r}=\frac{e^{\phi}\left(e^{\phi}-2\right) e^{-p \phi}}{e^{\phi}+1}+\frac{e^{(p+1) \phi}}{e^{\phi}+1}+\tanh \frac{\phi}{2}\left[e^{p \phi}-e^{\phi} \sum_{j=1}^{p-1} e^{-j \phi} s_{j}-\sum_{j=1}^{p-1} e^{j \phi} s_{j}\right]
$$


and eventually,

$$
\delta_{r}=\frac{2 e^{\frac{\phi}{2}} \cosh \frac{\phi}{2} L_{r}}{e^{2 k \phi}+e^{\phi}}=\frac{\left(e^{\phi}+1\right) L_{r}}{e^{2 k \phi}+e^{\phi}} .
$$

Now, we continue with the second step, i.e. summing together all the partial contributions. In order to do that, we are going to use two subscripts. The first subscript is $p$, which was defined earlier, while the second subscript $q$ distinguishes between bars of the same p-value. In the case of bars with $p<k$, we must evaluate

$$
\sum_{p=1}^{k-1} \sum_{q=1}^{2^{p-1}}\left[\left(\delta_{l}\right)_{p q}+\left(\delta_{r}\right)_{p q}\right]
$$

where

$$
\begin{gathered}
\left(\delta_{l}\right)_{p q}=\frac{2 e^{\frac{\phi}{2}} \cosh \frac{\phi}{2}\left(L_{l}\right)_{p q}}{e^{2 k \phi}+e^{\phi}} \quad 1 \leq p \leq k-1, \\
\left(\delta_{r}\right)_{p q}=\frac{2 e^{\frac{\phi}{2}} \cosh \frac{\phi}{2}\left(L_{r}\right)_{p q}}{e^{2 k \phi}+e^{\phi}} \quad 1 \leq p \leq k-1, \\
\left(L_{l}\right)_{p q}=\frac{e^{\phi}\left(e^{\phi}-2\right) e^{-p \phi}}{e^{\phi}+1}+\frac{e^{(p+1) \phi}}{e^{\phi}+1}+\tanh \frac{\phi}{2}\left[e^{p \phi}+e^{\phi} \sum_{j=1}^{p-1} e^{-j \phi} s_{q j}+\sum_{j=1}^{p-1} e^{j \phi} s_{q j}\right],
\end{gathered}
$$

and

$$
\left(L_{r}\right)_{p q}=\frac{e^{\phi}\left(e^{\phi}-2\right) e^{-p \phi}}{e^{\phi}+1}+\frac{e^{(p+1) \phi}}{e^{\phi}+1}+\tanh \frac{\phi}{2}\left[e^{p \phi}-e^{\phi} \sum_{j=1}^{p-1} e^{-j \phi} s_{q j}-\sum_{j=1}^{p-1} e^{j \phi} s_{q j}\right] .
$$

When we substitute the above equations into Eq. (6), the following quantity will appear:

$$
\begin{aligned}
\sum_{q=1}^{2^{p-1}}\left(L_{l}\right)_{p q} & =2^{p-1}\left[\frac{e^{\phi}\left(e^{\phi}-2\right) e^{-p \phi}}{e^{\phi}+1}+\frac{e^{(p+1) \phi}}{e^{\phi}+1}+e^{p \phi} \tanh \frac{\phi}{2}\right]+ \\
& +\sum_{q=1}^{2^{p-1}} \sum_{j=1}^{p-1}\left(e^{\phi} e^{-j \phi}+e^{j \phi}\right) s_{q j} \tanh \frac{\phi}{2}
\end{aligned}
$$

The same expression arises for $\left(L_{r}\right)_{p q}$, except a minus sign before the double sum. The double summation term is zero because, for a given $j, s_{q j}= \pm 1$ in equal numbers of bars. Hence:

$$
\sum_{p=1}^{k-1} \sum_{q=1}^{2^{p-1}}\left[\left(\delta_{l}\right)_{p q}+\left(\delta_{r}\right)_{p q}\right]=\frac{e^{\phi}+1}{e^{2 k \phi}+e^{\phi}} \sum_{p=1}^{k-1} \sum_{q=1}^{2^{p-1}}\left[\left(L_{l}\right)_{p q}+\left(L_{r}\right)_{p q}\right]=\frac{2^{k}\left[e^{(k-1) \phi}-e^{-(k-1) \phi}\right]}{1+e^{(2 k-1) \phi}} .
$$

Finally, the contribution from all bars with $p \geq k$ is

$$
\sum_{p=k}^{\infty} 2^{p-1}\left(h_{+}-h_{-}\right)=\sum_{p=k}^{\infty} 2^{p-1} 2\left(e^{\phi}-2\right) e^{-p \phi}=\frac{2^{k} e^{-k \phi}\left(e^{\phi}-2\right)}{1-2 e^{-\phi}} .
$$


Thus, $\operatorname{Esc}(k)$, the fraction of h-orbits in the interval $(-1,1)$ which escape after at least $\mathrm{k}$ bounces, is found by adding Eq. (7) and Eq. (8) and dividing by 2 :

$$
E s c(k)=\frac{1}{2}\left\{\frac{2^{k}\left[e^{(k-1) \phi}-e^{-(k-1) \phi}\right]}{1+e^{(2 k-1) \phi}}+\frac{2^{k} e^{-k \phi}\left(e^{\phi}-2\right)}{1-2 e^{-\phi}}\right\}=2^{k-1} \frac{e^{k \phi}\left(1+e^{\phi}\right)}{e^{\phi}+e^{2 k \phi}} .
$$

\section{B. Numerical Results}

In order to test our analytical result, we advanced Eq. (1) and (2) numerically. The interval $(-1,1)$ was divided into $\mathrm{N}$ subintervals, each having a width of $2 / N$. The initial conditions were

$$
X_{0}=-1+k \frac{2}{N}, \quad w_{0}=-\left(X_{0}-s_{0}\right) \tanh \frac{\phi}{2}, \quad k=1,2, \ldots, N,
$$

with the initial values $X_{0}$ distributed uniformly in the interval $(-1,1)$. Using the iterative scheme given by Eq. (1) and (2), $h$-orbits were evolved forward in time and for each $h$ orbit, we found the minimum number of iterations $k$ required to satisfy $\left|X_{k}\right|>1$. An escape distribution is built up by determining what fraction of the $h$-orbits escaped on the first bounce, on the second bounce and so on. The simulations were done for different values of $\phi$ and $N$. The value of $N$ was even increased to $N=5000000$ to ensure that the results were independent of the number of subdivisions. Fig.2 shows the escape distribution for different values of $\phi$, both analytical [Eq. (9)] and numerical .

The numerical results are in excellent agreement with those obtained from Eq. (9). There is only a small range of $e^{\phi}$ for which there is some discrepancy between the numerical and the analytical results for some p-bars. Every horizontal bar has a point $h_{D}$, which, according to [8], is given by

$$
h_{D}=\frac{2 \sinh \frac{\phi}{2}}{\cosh \left[\left(n-\frac{1}{2}\right) \phi\right]} \sum_{j=1}^{n-1} s_{j} \sinh [(n-j) \phi]
$$

and for which $X_{n}=0$. When $h_{-}+\delta_{r}>h_{D}$ or $h_{+}-\delta_{l}<h_{D}$, our analytical result needs some correction, i.e. the difference in bar length $h_{D}-\left(h_{-}+\delta_{r}\right)$ or $\left(h_{+}-\delta_{l}\right)-h_{D}$. For example, also visible in fig.2, for $e^{\phi}=3$ and $k=3$ our analytical result is around $3.5 \%$ larger than the numerical one and that happens because our calculation overestimates the contribution of the left side of the $p=2$ bar (for $h>0$; for $h<0$ is the right side of the $p=2$ bar that exhibits some problem). For $e^{\phi}=3.2$, the error for $k=3$ reduces to around $1 \%$. If the extra 
bar length is subtracted from our analytical calculation, we obtain the correct percentage. For the $p=2$ bar, the correction is:

$$
h_{D}-\left(h_{-}+\delta_{r}\right)=1-2\left(e^{-\phi}-e^{-2 \phi}\right)+\frac{1-2 e^{\phi}-e^{2 \phi}+2 e^{3 \phi}}{e^{\phi}+e^{6 \phi}}-\frac{1-e^{\phi}-e^{2 \phi}+e^{3 \phi}}{1+e^{3 \phi}} .
$$

\section{HILL'S PROBLEM AND H-ORBITS}

As it was stated in the introduction, Hénon's billiard model was inspired by [10], which dealt with the interaction of two satellites around a planet. That study was done in the context of the so called Hill's problem, a special case of the restricted three body problem where the massless particle moves in the neighbourhood of the secondary body. Originally intended as a model for the motion of the Moon around the Earth with perturbations by the sun, with some modification it can also serve as a simplified model of the dynamical behaviour of escaping stars in globular clusters [7]. In that case, the centre of the galaxy and the globular cluster play the role of the two main bodies, while the star is treated as the massless particle.

Let us assume that the cluster moves on a circular orbit of radius $R$ around the centre of the galaxy with constant angular velocity, the mass of the cluster with respect to the galaxy is assumed to be small, the mass of the star is considered to be negligible compared to that of the cluster and all three bodies lie in the same plane. If we consider a coordinate system that rotates with the angular velocity of the cluster around the galaxy and with its origin being at the cluster, Hill's equations can be written as follows [13]:

$$
\begin{aligned}
\ddot{\xi}-2 \dot{\eta}-3 \xi & =-\frac{\xi}{r^{3}} \\
\ddot{\eta}+2 \dot{\xi} & =-\frac{\eta}{r^{3}},
\end{aligned}
$$

where $\xi$ and $\eta$ are the $x$ and $y$ coordinates of the star and $r=\sqrt{\xi^{2}+\eta^{2}}$. Note that the above equations have been scaled in terms of time and distance. In the above mentioned coordinate system, the two Lagrangian points of interest occur at $\left(-\frac{1}{3} \frac{1}{3}^{3}, 0\right)$ and $\left(\frac{1}{3}^{\frac{1}{3}}, 0\right)$.

One recalls that in our inclined billiard model, the particle was dropped with zero horizontal velocity onto the inclined surface from a fixed height, i.e. with constant $\phi$ for various $h$ in the interval $(-1,1)$. In Hill's problem, the h-orbits can be defined to be those with initial conditions given by

$$
\xi_{0}=C, \quad \dot{\xi}_{0}=0, \quad \eta_{0}=0
$$


At a fixed initial value of the initial Hamiltonian $H_{i n}$ (analogous to the condition of constant $\phi$ in Hénon's model), $\dot{\eta}_{0}$ is calculated from

$$
H_{i n}=\frac{1}{2}\left(\dot{\xi}_{0}^{2}+\dot{\eta}_{0}^{2}\right)-\frac{3}{2} \xi_{0}^{2}-\frac{1}{\left|\xi_{0}\right|}
$$

Each time the orbit intersects the surface of section $\eta=0$ (with $\dot{\eta}>0$ when $\xi>0$ and with $\dot{\eta}<0$ when $\xi<0$ ), the value of $\xi$ is recorded. If $\xi=\xi_{j}$ on the $j$ th intersection with the surface of section, then the orbit may be represented symbolically by a sequence $\left\{d_{j}, j=1 \ldots \infty\right\}$, where

$$
d_{j}= \begin{cases}0 & \text { if } \xi_{j}<0 \\ 1 & \text { if } \xi_{j}>0\end{cases}
$$

If the orbit escapes through the right Lagrangian point after $k$ intersections with the surface of section, then $d_{j}=1, j>k$. Similarly, if the orbit escapes through the left Lagrangian point after $k$ intersections with the surface of section, then $d_{j}=0, j>k$. Hence a real number can be attached to a given orbit in a similar way as it was done for the inclined billiard model.

Fig. 3 is an example of an h-orbit in Hill's problem, exhibiting similar features to those found in fig. 1 .

\section{SUMMARY}

Hénon [8] used an inclined billiard in order to investigate the phenomenon of chaotic scattering. A point particle, initially at rest, fell from a certain height and bounced elastically off the surface of two disks. When the particle bounced beyond the top of either disk, it was considered to have escaped. By assigning a number $A$ to the orbit of the particle, we were able to plot $A(h)$, where $h(-1 \leq h \leq 1)$ was the initial value of the $\mathrm{x}$-coordinate of the particle. For certain values of the parameter $\phi$, which was related to the particle energy, the graphical representation of $A(h)$ had the form of the so-called Devil's staircase. Based on certain properties of the Devil's staircase, we were able to derive an analytical expression for the number of particles that escaped after at least $k$ bounces.

It may be possible to approach the issue of stars escaping from a cluster or other similar situations by using the billiard model as a simplification of Hill's problem. Therefore, our future aim is to investigate the possibility of connecting the billiard model and Hill's problem in a way that can be used to describe a more complex situation such as for example the 
dynamical evolution of a star within a globular cluster. The latter is far more complicated as a system than the inclined billiard we have studied, but many times, it is possible to get a good approximation of a complicated system by following a more simple approach to the problem.

\section{ACKNOWLEDGEMENTS}

The authors would like to thank Professor D.C. Heggie for valuable comments regarding certain aspects of this work. 


\section{REFERENCES}

1. Astakhov, S.A., Lee, E.A., Farrelly, D., Formation of Kuiper-belt binaries through multiple chaotic scattering encounters with low-mass intruders, MNRAS, 2005, vol. 360, pp. 401-415.

2. Baumgardt, H., Scaling of N-body calculations, MNRAS, 2005, vol. 325, pp. 1323-1331.

3. Ernst, A., Just, A., Spurzem, R., Porth O., Escape from the vicinity of fractal basin boundaries of a star cluster, MNRAS, 2008, vol. 383,pp. 897-906.

4. Ford, E.B., Lystad, V., Rasio, F.A., Planet-planet scattering in the upsilon Andromedae system, Nature, 2005, vol. 434, pp. 873-876.

5. Fukushige, T., Heggie, D.C., The time-scale of escape from star clusters, MNRAS, 2000, vol. 318 , pp. $753-761$.

6. Heggie, D.C., Mass loss from globular clusters, Dynamics of Star Clusters and the Milky Way, S. Deiters, B. Fuchs, A. Just, R. Spurzem, R. Wielen (Eds.), San Francisco: Astron. Soc. Pac., 2001, ASP Conf. Ser. vol. 228, pp. 29-41.

7. Heggie, D.C., Escape in Hill's problem, The restless universe, Proceedings of the 54th Scottish Univ. Summer School in Physics, B.A. Steves, A.J. Maciejewski (Eds.), Bristol: Scottish Universities Summer School in Physics and IoP Publishing, 2001, pp. 109-128.

8. Hénon, M., Chaotic scattering modelled by an inclined billiard, Physica D, 1988, vol. 33, pp. $132-156$.

9. Leon, S., Meylan, G., Combes, F., Tidal tails around 20 Galactic globular clusters. Observational evidence for gravitational disk/bulge shocking, A\&A, 2000, vol. 359, pp. 907-931.

10. Petit, J.M., Hénon, M., Satellite encounters, Icarus, 1986, vol. 66, pp. 536-555.

11. Petit, J.M., Chaotic scattering in planetary rings, Singularities in gravitational systems, D. Benest, C. Froeschl (Eds.), Germany: Springer, 2002, Lect. Not. Phys. vol. 590, pp. 114-144.

12. Press, W.H., Teukolsky, S.A., Vetterling, W.T., Flannery, B.P., Numerical Recipes In Fortran 77. Second edition. New York: Cambridge Univ. Press, 1996.

13. Szebehely, V.G., Theory of orbits, New York: Academic Press, 1967. 


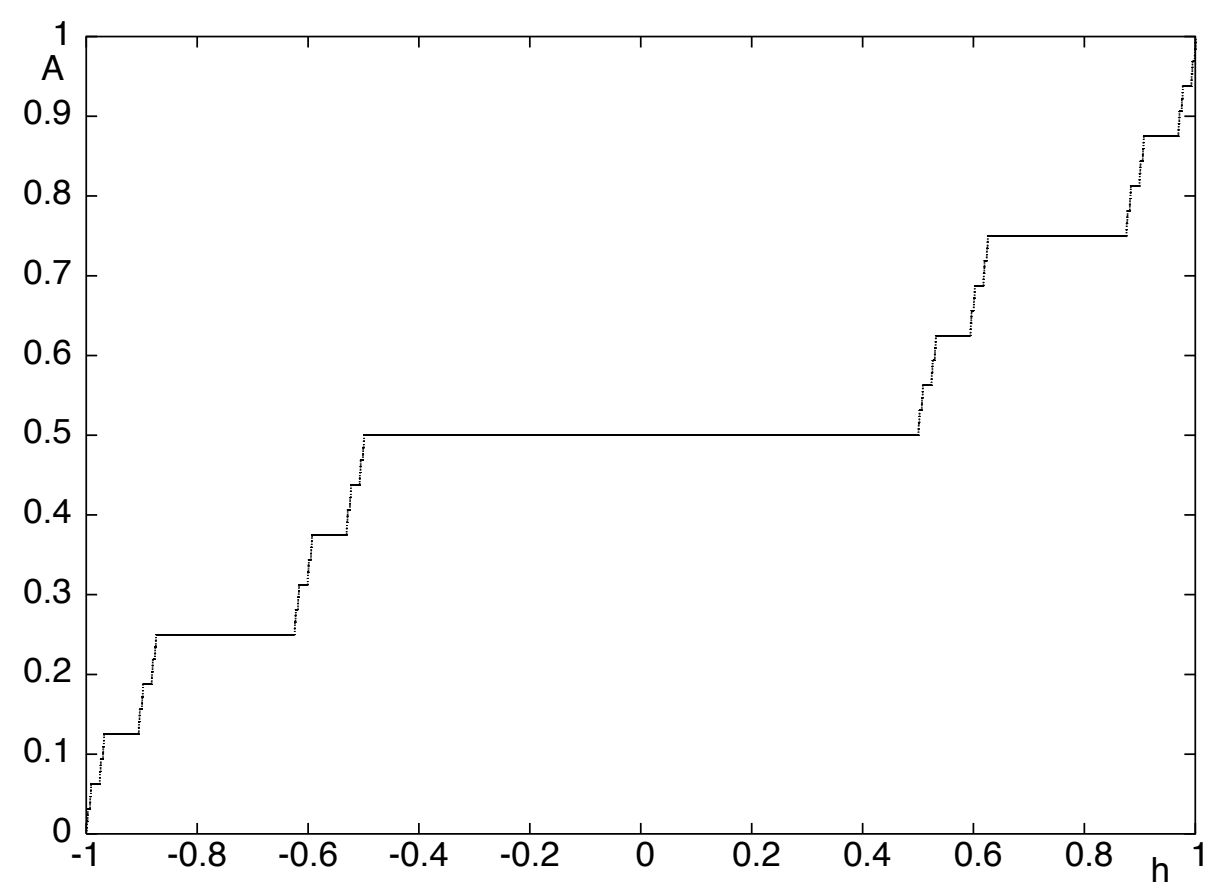

FIG. 1:The Devil's staircase for $e^{\phi}=4$. 


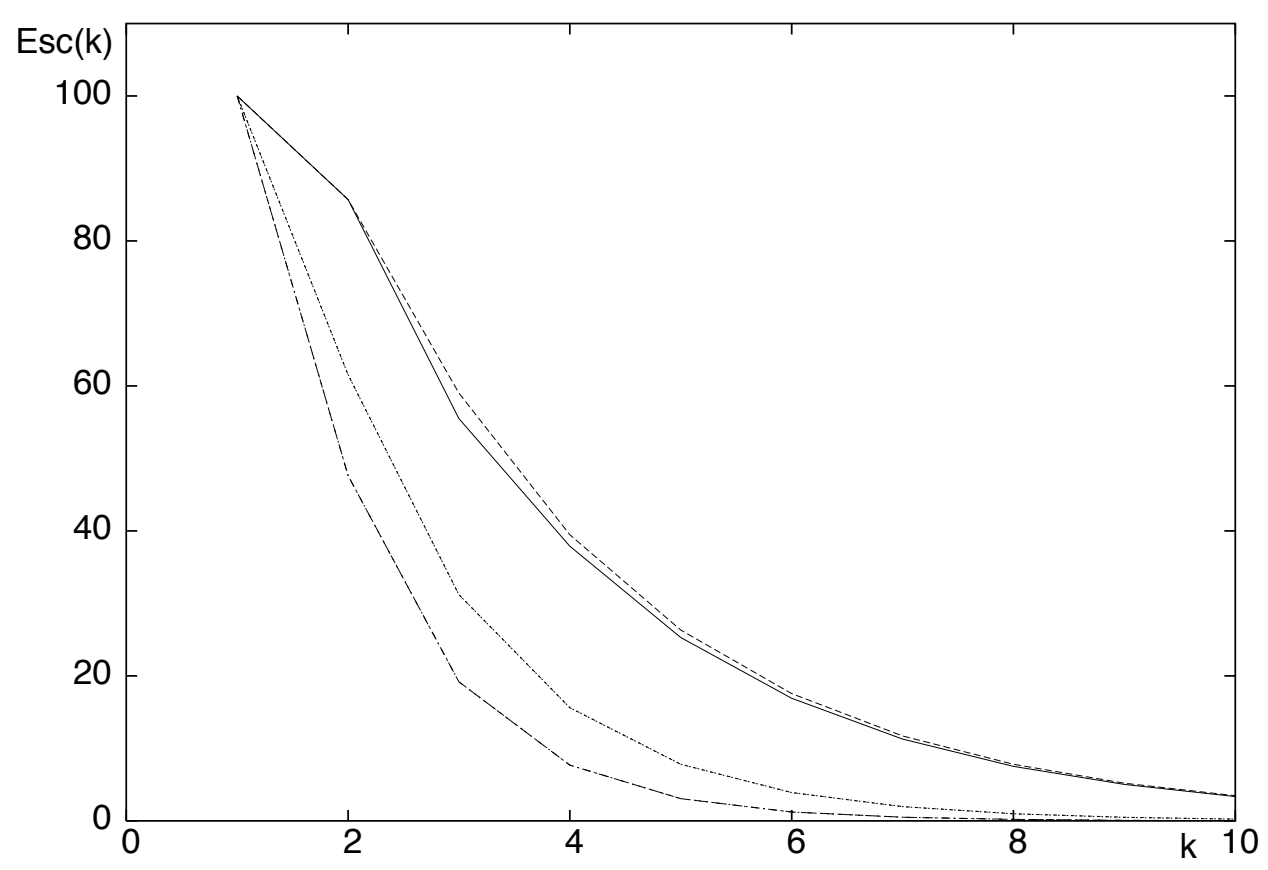

FIG. 2:Percentage of particles that escape after at least $k$ bounces against the number of bounces $k$. From right to left, the first two curves correspond to $e^{\phi}=3$ (the left curve comes from the simulations, while the right one is based on equation 28), the third one corresponds to $e^{\phi}=4$ and the fourth one corresponds to $e^{\phi}=5$. Note that each of the third and fourth curves are actually two curves (one on top of the other one), as the numerical and analytical results are almost identical. Also note the small discrepancy between the numerical and analytical results for $e^{\phi}=3$. 


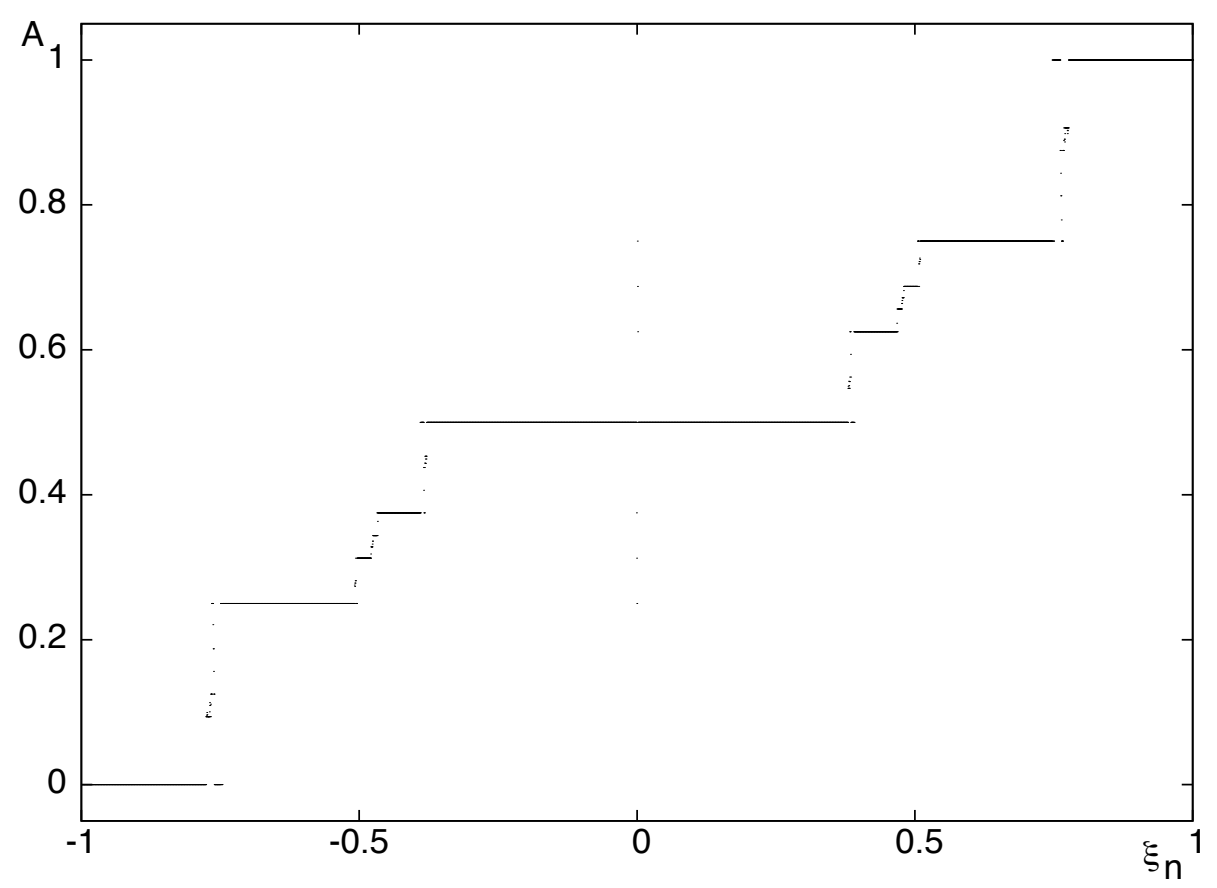

FIG. 3:Orbital sequence number $A$ against $\xi_{n}$ in Hill's problem, where $\xi_{n}=\frac{1}{3}^{-\frac{1}{3}} \xi_{0}$. The initial value of the Hamiltonian is $H_{\text {in }}=-1.9$. The results for Hill's problem were obtained by integrating equations (10) and (11) numerically, using a Burilsch-Stoer integrator with a variable time step [12]. The time of integration was $4 \pi$ which, in our system of units, is twice the orbital period of the cluster around the galaxy. 